REVISTA PROYECCIONES N ${ }^{\circ}$ 8: 15-59.

Diciembre 1984 - ISSN 0716-0917

JORNADA MATEMATICAS, Agosto 1984

\title{
TEORÍA DE OPERADORES Y APLICACIONES
}

\section{Dr. VICTOR CORTES*}

\section{INTRODUCCION .}

Estas notas reúnen el material necesario para desarrollar la teoría espectral para operadores tanto acotados como no acotados, se ha trabajado un sinnúmero de ejemplos, en los cuales se visualiza las aplicaciones de los teoremas y proposiciones. Los tópicos cubiertos en este cursillo son un resumen parcial del seminario "Tópicos de Análisis", dic tado por el autor en la Facultad de Ciencias Básicas de la Universidad de Chile, durante el año 1983.

Dentro de la Teoría Espectral, el problema que nos interesa pre sentar aquí es el de la equivalencia unitaria entre operadores autoadjun tos y los operadores multiplicación en $\mathcal{L}^{2}(\mathrm{M}, \mu)$, siendo nuestros objetivos finales el teorema 5.6. del primer Capítulo y el teorema 2.3. del segundo.

Los prerrequisitos para poder seguir estas notas, son los cursos básicos de Análisis y Ecuaciones Diferenciales. Se ha tratado de que al terminar estas notas, el lector posea el conocimiento necesario para leer las referencias y artículos dados en la sección 3 del Capítulo II. Ellos dan una idea del estado actual de la teoría y sus proyecciones.

* Profesor Departamento de Matemáticas, Facultad de Ciencias Básicas, Universidad de Chile. 
CAPITULO I. OPERADORES ACOTADOS.

Sean $B_{1}$ y $B_{2}$ espacios de Banach con normas \|\|$_{1}$ y \|\|$_{2}$ respectivamente. Supondremos siempre que $B_{1} \quad y \quad B_{2}$ son $\mathbb{C}$-espacios vecto riales.

Definición 0.1. Diremos que una aplicación $T: B_{1} \rightarrow B_{2}$ es un operador lineal acotado si y sólo si para todo $f, g \in B_{1} y \quad \alpha \in \mathbb{C}$ se tiene:

$$
T(f+g)=T(f)+T(g)
$$

$$
T(\alpha f)=\alpha T(f)
$$

$$
\sup \left\{\|\mathrm{Tf}\|_{2}: \mathrm{f} \in \mathrm{B}_{1},\|\mathrm{f}\|_{1}=1\right\}<\infty .
$$

Denotaremos por $\mathcal{L}\left(\mathrm{B}_{1}, \mathrm{~B}_{2}\right)$ al espacio vectorial de tales operadores donde la suma y producto por escalar se definen como siempre

$$
\begin{aligned}
\left(T_{1}+T_{2}\right)(f) & =T_{1}(f)+T_{2}(f) \\
\left(\alpha T_{2}\right)(f) & =\alpha T_{2}(f) .
\end{aligned}
$$

En $\mathcal{L}\left(\mathrm{B}_{1}, \mathrm{~B}_{2}\right)$ podemos definir una norma de la manera siguiente; para $T \in \mathcal{L}\left(B_{1}, B_{2}\right)$

$$
\|T\|=\sup \left\{\|T f\|_{2}: E \in B_{1} \quad y \quad\|f\|_{1}=1\right\} .
$$


Con esta norma $\mathcal{L}\left(\mathrm{B}_{1}, \mathrm{~B}_{2}\right)$ resulta ser un espacio normado completo, es decir, un espacio de Banach. Para simplificar la notación denotaremos por $\mathcal{L}(\mathrm{H})$ al conjunto de operadores lineales acotados de $\mathrm{H}$ en $\mathrm{H}$ donde $\mathrm{H}$ es un espacio de Hilbert.

Definición 0.2. Sea B un espacio de Banach. Definimos su dual, denotado por $B^{*}$, como $B^{*}=\mathcal{L}(B, \mathbb{C})$. $B^{*}$ es un espacio de Banach y a sus elementos se les llama funcionales lineales acotados.

§ 1. Topologías en $\mathcal{L}\left(\mathrm{B}_{1}, \mathrm{~B}_{2}\right)$.

A la topología inducida por la norma \|\| en $\mathcal{L}\left(\mathrm{B}_{1}, \mathrm{~B}_{2}\right)$ se le $11 \mathrm{a}-$ ma la topología uniforme de operadores (o topología de la norma). En esta topología la aplicación

$$
P: \mathcal{L}\left(\mathrm{B}_{1}, \mathrm{~B}_{2}\right) \times \mathcal{L}\left(\mathrm{B}_{2}, \mathrm{~B}_{3}\right) \rightarrow \mathcal{L}\left(\mathrm{B}_{1}, \mathrm{~B}_{3}\right)
$$

definida por $\mathrm{P}(\mathrm{A}, \mathrm{B})=\mathrm{BA}$ es contínua. Una sucesión $\left\{\mathrm{T}_{\mathrm{n}}\right\}$ de operadores en $\mathcal{L}\left(B_{1}, B_{2}\right)$ converge a $T$ en la topología uniforme de operadores si y sólo si $\left\|T_{n}-T\right\|$ converge a cero cuando $n$ tiende a infinito. Introduciremos dos topologías más en $\mathcal{L}\left(\mathrm{B}_{1}, \mathrm{~B}_{2}\right)$.

Definición 1.1. La topología fuerte de operadores es la topología más débil en $\mathcal{L}\left(\mathrm{B}_{1}, \mathrm{~B}_{2}\right)$ tal que

$$
E_{f}: \mathcal{L}\left(B_{1}, B_{2}\right) \rightarrow B_{2}
$$


dada por $E_{f}(T)=T(f)$ son aplicaciones contínuas para todo $f \in B_{1}$. En esta topología una sucesión (o más generalmente una red) de operadores $\left\{\mathrm{T}_{n}\right\}_{n=1}^{\infty}$ en $\mathcal{L}\left(\mathrm{B}_{1}, \mathrm{~B}_{2}\right)$ converge a $T$ sí y sólo si $\left\|\mathrm{T}_{\mathrm{n}} \mathrm{f}-\mathrm{Tf}\right\|_{2}$ converge a cero cuando $n$ tiende a infinito para todo $f \in B_{1}$. Denotaremos tal límite como $\underset{n}{S-1 i ́ m} T_{n}=T$ o $T_{n} \stackrel{S}{\longrightarrow}$. La aplicación $P$ definida anteriormente resulta ser contínua en cada variable (lo cual no implica continuidad de P).

Definición 1.2. La topología débil de operadores en $\mathcal{L}\left(\mathrm{B}_{1}, \mathrm{~B}_{2}\right)$ es la topología más débil tal que las aplicaciones

$$
E_{f, l}: \mathcal{L}\left(B_{1}, B_{2}\right) \rightarrow \mathbb{C}
$$

dadas por $E_{f, l^{(T)}}=\ell(T f)$ son contínuas para todo $f \in B_{1}$ y $\quad l \in B_{2}^{*}$. Una sucesión (o red) de operadores $\left\{\mathrm{T}_{\mathrm{n}}\right\}_{\mathrm{n}=1}^{\infty}$ converge a $T$ en esta topología sí y sólo si

$$
\left|\ell\left(T_{n}(f)\right)-\ell(T(f))\right| \rightarrow 0
$$

cuando $n$ tiende a infinito para todo $\ell \in B_{2}^{*}$ y $f \in B_{1}$. En este caso la convergencia la denotaremos por $\underset{w}{ }-1 i_{n} T_{n}=T$ o $T_{n} \stackrel{w}{\longrightarrow} T$.

Es interesante hacer notar que en el caso de $\mathcal{L}(\mathrm{H}), \mathrm{H}$ espacio de Hilbert, la topología débil de operadores es equivalente a

$$
\mathrm{w}-\lim \mathrm{T}_{\mathrm{n}}=\mathrm{T} \Leftrightarrow \lim _{\mathrm{n}}\left\langle\mathrm{T}_{\mathrm{n}} \mathrm{f}, \mathrm{g}\right\rangle=\langle\mathrm{Tf}, \mathrm{g}\rangle
$$

para todo $f, g \in H$. Esto es inmediato del teorema de representación de 
Reiz, que dice que todo funcional $l \in \mathrm{H}^{*}$ se representa unívocamente por

$$
\ell(f)=\langle f, g\rangle
$$

con $g$ en $\mathrm{H}$.

Examinaremos algunos ejemplos que nos ayudarán a visualizar la diferencia entre estas topologías.

Ejemplos: Consideremos el espacio de Hilbert $\ell_{2}(\mathbb{C})$, es decir $\ell_{2}(\mathbb{C})=\left\{\left\{x_{i}\right\}_{i=1}^{\infty}: x_{i} \in \mathbb{C}, \sum_{i=1}^{\infty}\left|x_{i}\right|^{2}<\infty\right\}$. El producto interno está definido por

$$
\left\langle\left\{x_{i}\right\}_{i=1}^{\infty},\left\{y_{i}\right\}_{i=1}^{\infty}\right\rangle=\sum_{i=1}^{\infty} x_{i} \bar{y}_{i}
$$

y luego la norma de un elemento $\left\{\mathrm{x}_{i}\right\}_{i=1}^{\infty}$ está dada por

$$
\left\|\left\{x_{i}\right\}\right\|_{\ell_{2}}=\left(\sum_{i=1}^{\infty}\left|x_{i}\right|^{2}\right)^{1 / 2}
$$

Definamos en $\ell_{2}$ (C) las siguientes sucesiones de operadores lineales y acotados:

$$
\begin{aligned}
& T_{n}\left(x_{1}, x_{2}, \ldots\right)=\left(\frac{1}{n} x_{1}, \frac{1}{n} x_{2}, \ldots\right) \\
& S_{n}\left(x_{1}, x_{2}, \ldots\right)=(\underbrace{0, \ldots, 0}_{n}, x_{n+1}, x_{n+2}, \ldots) \\
& W_{n}\left(x_{1}, x_{2}, \ldots\right)=(\underbrace{0, \ldots, 0}_{n}, x_{1}, x_{2}, \ldots) .
\end{aligned}
$$


Puesto que $\underset{n}{\lim }\left\|T_{n}-0\right\|=\lim _{n} \frac{1}{n}=0$ entonces $\left\{T_{n}\right\}_{n=1}^{\infty}$ converge en la topología uniforme de operadores al operador cero.

También es claro que si $f=\left\{x_{i}\right\}_{i=1}^{\infty}$ entonces

$$
\lim _{n}\left\|s_{n} f-o\right\|_{\ell}^{2}=\lim _{n} \sum_{k=n+1}^{\infty}\left|x_{k}\right|^{2}=0
$$

debido a que $\sum_{k=1}^{\infty}\left|\mathrm{x}_{\mathrm{k}}\right|^{2}<\infty$; por lo tanto hemos demostrado que s-lím $S_{n}=0$. Ahora probaremos que el límite de $s_{n}$ en la topología de la norma no es cero. Para ver esto basta tomar $\mathrm{E}_{\mathrm{n}}=\left\{\mathrm{x}_{i}\right\}_{i=1}^{\infty}$ tal que $x_{i}=0 \quad \forall i \neq n+1$ y $x_{n+1}=1$. Entonces

$$
1=\left\|S_{n} f_{n}\right\|_{\ell_{2}} \leqslant\left\|_{n}-O\right\|_{\mathcal{L}(H)}
$$

y luego $S_{n}$ no puede converger a cero en la topología de la norma.

En forma similar dejamos como ejercicio al lector el demostrar que $\mathrm{W}_{\mathrm{n}}$ converge a cero en la topología débil de operadores y que $W_{n}$ no converge en la topología uniforme ni en la topología fuerte de operadores.

5 2. Operador adjunto.

En esta sección definiremos el operador adjunto de un operador acotado en un espacio de Banach y en un espacio de Hilbert. Estas dos definiciones no coinciden aunque están intimamente ligadas.

Definición 2.1. Sean $B_{1}$ Y $B_{2}$ espacios de Banach, $T$ un operador 1ineal y acotado de $B_{1}$ en $B_{2}$. El adjunto de $T$, el cual denotaremos 
por $T^{\prime}$, es el operador lineal y acotado de $B_{2}^{*}$ en $B_{1}^{*}$ definido por

$$
\left(T^{\prime} \ell\right)(f)=\ell(T f)
$$

para todo $l \in B_{2}^{*} \quad y \quad f \in B_{1}$.

Ejemplo: Sea $B_{1}=l_{1}=B_{2}$ donde $l_{1}$ es el conjunto de las sucesiones de números complejos tales que su serie es absolutamente convergente. El dual de $\ell_{1}$ es $l_{\infty}$, las sucesiones acotadas. Si $T$ es el operador "corrimiento"

$$
T\left(x_{1}, x_{2}, \ldots\right)=\left(0, x_{1}, x_{2}, \ldots\right)
$$

entonces es fácil ver que su dual $T^{\prime}: \ell_{\infty} \rightarrow l_{\infty}$ está definido por

$$
T^{\prime}\left(x_{1}, x_{2}, \ldots\right)=\left(x_{2}, x_{3}, \ldots\right)
$$

Nótese que en este ejemplo $\|T\|=1=\left\|T^{\prime}\right\|$. Más aún, este hecho es general para los operadores adjuntos.

Lema 2.2. Sean $\mathrm{B}_{1}, \mathrm{~B}_{2}$ espacios de Banach $\mathrm{Y} \quad \mathrm{T}: \mathrm{B}_{1} \rightarrow \mathrm{B}_{2}$ un operador lineal y acotado. Entonces:

$$
\|T\|_{\mathcal{L}\left(B_{1}, B_{2}\right)}=\left\|T^{\prime}\right\|_{\mathcal{L}\left(B_{2}^{*}, B_{1}^{*}\right)}
$$

Demostración: Usando uno de los corolarios del teorema de Hahn-Banach [3] se tiene que:

$$
\|\mathrm{TE}\|_{2}=\sup _{\|\ell\| \leq 1}|\ell(\mathrm{Tf})|, \quad \ell \in \mathrm{B}_{2}^{*}
$$

luego, 


$$
\begin{aligned}
\|T\|_{\mathcal{L}\left(B_{1}, B_{2}\right)} & =\sup _{\|f\| \leq 1}\|T f\|_{2}=\sup _{\|f\| \leq 1} \sup _{\|\ell\| \leq 1}|\ell(T f)| \\
& =\sup _{\|\ell\| \leq 1}\left(\sup _{\|f\| \leq 1}\left|\left(T^{\prime} \ell\right)(f)\right|\right) \\
& =\sup _{\|\ell\| \leq 1}\|T \cdot \ell\|=\left\|T^{\prime}\right\|_{\mathcal{L}\left(B_{2}^{*}, B_{1}^{*}\right)} .
\end{aligned}
$$

Para el caso de un espacio de Hilbert procederemos de la siguiente manera:

Definición 2.3. Sea $H$ un espacio de Hilbert $y \quad T: H \rightarrow H$ un operador lineal y acotado. Al operador $A: H \rightarrow H$ tal que

$$
\langle\mathrm{Tf}, \mathrm{g}\rangle=\langle\mathrm{f}, \mathrm{Ag}\rangle
$$

se le llama el operador adjunto de $T$ Y lo denotaremos por $A=T^{*}$. No es difícil demostrar que $T^{*}$ es un operador lineal y acotado. Una pregunta nace de inmediato: ¿Qué relación existe entre $T^{*}$ y $T^{\prime}$ en el caso de un espacio de Hilbert?, (que en particular es un espacio de Banach).

Proposición 2.4. Sea $\mathrm{H}$ un espacio de Hilbert $\mathrm{y}$ T : $\mathrm{H} \rightarrow \mathrm{H}$ un operador lineal $y$ acotado. Definamos $C: H \rightarrow H^{*}$ por $C(g)(f)=\langle f, g\rangle$. Entonces $T^{*}=C^{-1} T^{\prime} C$.

Demostración: Por definición de $T^{\prime}$ y C se obtiene que

$$
\langle T f, g\rangle=C(g)(T f)=T^{\prime} C(g)(f)
$$


luego

$$
\langle T f, g\rangle=C\left(C^{-1} T^{\prime} C\right)(g)(f)=\left\langle f, C^{-1} T^{\prime} C g\right\rangle
$$

y usando la unicidad de $T^{*}$ obtenemos que $T^{*}=C^{-1} T^{\prime} C$.

El próximo teorema enumera algunas propiedades de los operadores adjuntos. La demostración se deja como ejercicio al lector.

Teorema 2.5. Sea $\mathrm{H}$ espacio de Hilbert. Entonces

i) $\quad *: \mathcal{L}(\mathrm{H}) \rightarrow \mathcal{L}(\mathrm{H})$ es un isomorfismo isométrico conjugado

ii) $\quad(\mathrm{TS})^{*}=\mathrm{S}^{*} \mathrm{~T}^{*}$

iii) Si $T^{-1}$ existe entonces $T^{*}$ es invertible $y \quad\left(T^{*}\right)^{-1}=\left(T^{-1}\right)^{*}$

iv) $\quad\left(T^{*}\right)^{*}=T$

v) *: $\mathscr{L}(\mathrm{H}) \rightarrow \mathcal{L}(\mathrm{H}), \mathrm{T} \rightarrow \mathrm{T}^{*}$ es contínua en la topología débil y uniforme de operadores.

vi) $\quad\left\|T^{*} T\right\|=\|T\|^{2}$

Es interesante hacer notar que * : $\mathcal{L}(\mathrm{H}) \rightarrow \mathcal{L}(\mathrm{H})$ no es necesaria mente contínua con respecto a la topología fuerte de operadores.

Ejercicios: a) Dé un ejemplo mostrando que la continuidad de * en $\mathcal{L}(\mathrm{H})$ no se cumple necesariamente para $\mathrm{H}$ de dimensión infinita. b) * : $\mathcal{L}(\mathrm{H}) \rightarrow \mathscr{L}(\mathrm{H}) \quad$ es contínua en la topología fuerte de operadores sí y sólo si H es de dimensión finitiva.

Ahora definiremos una clase particular de operadores, los 11amados operadores autoadjuntos. Ellos juegan un papel muy importante en 
Análisis Funcional y en Física Matemática.

Definición 2.6. Un operador $T \in \mathcal{L}(\mathrm{H})$ es llamado autoadjunto $\mathrm{si} T=\mathrm{T}^{*}$.

Daremos ahora algunos ejemplos de operadores autoadjuntos.

Ejemplo: $\quad T: \mathbb{C}^{n} \rightarrow \mathbb{C}^{n}$ una función lineal definida en el espacio de Hilbert $\mathbb{C}^{\mathrm{n}}$. Es un fácil ejercicio de álgebra lineal demostrar que una función lineal en un espacio de dimensión finita es siempre acotada. Podemos representar a $T$ como una matriz de $n \times n$ con coeficientes complejos. $T$ es autoadjunto sí y sólo si la matriz tiene la forma $a_{i j}=\bar{a}_{j i}$ para $i \neq j, i, j=1, \ldots, n$, es decir

$$
\left(\begin{array}{llll}
a_{11} & a_{12} & \cdots \cdots & a_{1 n} \\
a_{21} & a_{22} & \cdots & a_{2 n} \\
\cdot & & & \cdot \\
a_{n 1} & & & a_{n n}
\end{array}\right)
$$$$
\text { con } a_{12}=\overline{a_{21}}, \ldots, a_{1 n}=\overline{a_{n 1}} \text {, etc. }
$$

Por ejemplo, la matriz

$$
\left(\begin{array}{rr}
1 & -i \\
i & i
\end{array}\right)
$$

representa un operador autoadjunto $T: \mathbb{C}^{2} \rightarrow \mathbb{C}^{2}$.

Ejemplo: Sea $\mathcal{L}^{2}([0,1])$ el espacio de Hilbert de las funciones de cua drado integrable en $[0,1]$. Definamos $T: \mathcal{L}^{2}([0,1]) \rightarrow \mathcal{L}^{2}([0,1])$ por 


$$
(T f)(x)=\varphi(x) f(x)
$$

donde $\varphi$ es una función acotada (o más generalmente esencialmente acotada) en $[0,1]$. Es fácil demostrar que $T$ es un operador lineal y acotado; $T$ se denota por $\mathrm{T}_{\varphi}$.

Entonces es claro que

$$
\begin{aligned}
& \langle T f, g\rangle=\int_{0}^{1} \Psi(x) E(x) \overline{g(x)} d x \\
& \langle f, T g\rangle=\int_{0}^{1} f(x) \overline{\psi(x)} \overline{g(x)} d x
\end{aligned}
$$

Luego $\mathrm{T}_{\varphi}$ es autoadjunto sí y sólo si $\varphi$ es una función a valores reales, es decir, $\overline{\varphi(x)}=\varphi(x)$ para todo $x \in[0,1]$.

\section{3. Proyectores y operadores unitarios.}

Una de las propiedades geométricas importantes de los espacios de Hilbert es que podemos definir el concepto de proyección y de unitariedad en forma simple.

Definición 3.1. Sea $M$ un subespacio de $\mathrm{H}$. Definimos el complemento ortogonal de $M$ como el conjunto $M^{\perp}$ en $H$ definido por

$$
M^{\perp}=\{f \in H:\langle f, g\rangle=0, \quad \forall g \in M\} .
$$

No es difícil demostrar que todo elemento $f \in H$ se representa en forma única como $f=g+h$ donde $g \in M \quad y \quad h \in M^{\perp}$, o equivalentemente $\mathrm{H}=\mathrm{M} \oplus \mathrm{M}^{\perp}$. El operador $\mathrm{P}_{\mathrm{M}}$ definido por $\mathrm{P}_{\mathrm{M}}(\mathrm{f})=\mathrm{g}$ es llamado un proyector. 
Definición 3.2. Sea $\mathrm{P} \in \mathcal{L}(\mathrm{H})$. Diremos que $\mathrm{P}$ es un proyector (o una proyección) sí y sólo si $\mathrm{P}^{2}=\mathrm{P}$. Si además $\mathrm{P}^{*}=\mathrm{P}$ entonces diremos que $P$ es un proyector ortogonal.

Definición 3.3. Sean $\mathrm{H}_{1}$ y $\mathrm{H}_{2}$ dos espacios de Hilbert. Diremos que

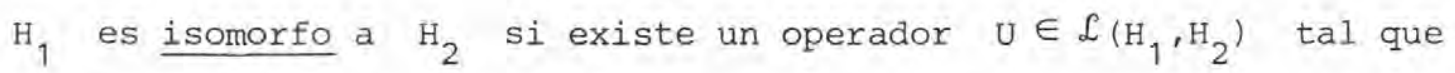
$\langle\mathrm{Ux}, \mathrm{Uy}\rangle_{\mathrm{H}_{2}}=\langle\mathrm{x}, \mathrm{y}\rangle_{\mathrm{H}_{1}}$ para todo $\mathrm{x}, \mathrm{y} \in \mathrm{H}_{1}$. Tal operador $\mathrm{U}$ se llama un operador unitario.

El próximo teorema caracteriza los proyectores.

Teorema 3.4. Sea $M$ un subespacio cerrado de $H$ y $P \in \mathcal{L}(H)$. Entonces i) $\quad \mathrm{M}^{\perp}$ es cerrado

ii) $\quad \mathrm{P}_{\mathrm{M}}$ es un proyector ortogonal

iii) P es un proyector sí y sólo si existe un subespacio cerrado M de $H$ tal que $P=P_{M}$.

Demostración: Las dos primeras partes son obvias y se basan en la continuidad del producto interno y en la definición del proyector $\mathrm{P}_{\mathrm{M}}$. Para la ultima parte, basta demostrar que Ran P es un subespacio cerrado de $H \quad Y$ que $M=\operatorname{Ran} P$.

Definiremos ahora el concepto de equivalencia unitaria entre operadores.

Definición 3.5. Sean $\mathrm{T}_{1} \quad \mathrm{Y} \quad \mathrm{T}_{2}$ operadores en $\mathcal{L}(\mathrm{H})$. Diremos que $\mathrm{T}_{1}$ es unitariamente equivalente a $\mathrm{T}_{2}$ sí y sólo si existe un operador unitario $\mathrm{U}$ tal que $\mathrm{T}_{1}=\mathrm{U}^{-1} \mathrm{~T}_{2} \mathrm{U}$. 
Ejemplos: a) Un operador multiplicación $T_{\varphi}$ en $\mathcal{L}^{2}([0,1])$ es un proyector ortogonal sí y sólo si $\varphi$ es una función real y $\varphi \cdot \psi=\varphi$. Luego $\varphi$ debe ser necesariamente una función característica sobre un subconjun to medible de $[0,1]$.

b) Un ejemplo de operador unitario es la transformada de Fourier en $\mathcal{L}^{2}(\mathbb{R})$. Es sabido de análisis que la transformada de Fourier satisface la ecuación (fórmula de Plancherel)

$$
\langle\hat{f}, \hat{g}\rangle_{\mathcal{L}^{2}(\mathbb{R})}=\langle f, g\rangle_{\mathcal{L}^{2}(\mathbb{R})} .
$$

c) Dejamos como ejercicio encontrar los operadores multiplicación que son operadores unitarios de $\mathcal{L}\left(\mathcal{L}^{2}([0,1])\right)$. También es interesante caracterizar los operadores unitarios en el caso finito. ¿Qué clase de matrices son?

\$ 4. Teoría espectral para operadores acotados.

Los valores propios de un operador lineal $T: \mathbb{C}^{n} \rightarrow \mathbb{C}^{n}$ son aquellos números complejos $\lambda$ tales que $\operatorname{det}(\lambda I-T)=0$. El conjunto de ta les valores propios es llamado el espectro del operador $T$. Puesto que $\operatorname{det}(\lambda I-T)$ es un polinomio de grado $n$, tiene a lo más $n$ raíces y por lo tanto el espectro $\sigma(T)$ de $T$ es un subconjunto finito de $\mathbb{I}$. Además si $\lambda$ no es un valor propio, el operador $(\lambda I-T)$ posee una in versa. Ahora generalizaremos tal concepto para el caso de un operador en $\mathcal{L}(\mathrm{H})$ donde $\mathrm{H}$ es un espacio de Hilbert de dimensión cualquiera.

Definición 4.1. Sea $T$ un operador en $\mathcal{L}(H)$. Un número complejo $\lambda$ pertenece al resolvente de $T, \rho(T)$, si $\lambda I-T$ posee una inversa 
acotada. Si $\lambda \notin \rho(T)$ diremos que $\lambda$ está en el espectro de $T$, el cual denotaremos por $\sigma(T)$. Además denotaremos por $R_{\lambda}(T)$ el operador inverso de $(\lambda I-T)$ para todo $\lambda$ en $P(T)$.

Teorema 4.2. Sea $T$ un operador en $\mathcal{L}(\mathrm{H})$. Entonces

i) $\quad \rho(T)$ es un subconjunto abierto de $\mathbb{C}$

ii) Para todo $\lambda, \mu \in \rho(T)$ se tiene que $R_{\lambda}(T)$ y $R_{\mu}$ (T) conmutan $y$

$$
R_{\lambda}(T)-R_{\mu}(T)=(\mu-\lambda) R_{\mu}(T) R_{\lambda}(T) .
$$

Demostración: i) Sea $\mu \in \rho(T)$. Afirmamos que si $\lambda$ es tal que $|\lambda-\mu|<\left\|\mathrm{R}_{\mu}(\mathrm{T})\right\|^{-1}$ entonces $\lambda \in \rho(\mathrm{T}) . \quad \mathcal{L}(\mathrm{H})$ es un espacio de Banach con la norma uniforme, luego toda serie absolutamente convergente es convergente. Usando este hecho es fácil demostrar que

$$
\tilde{R}_{\lambda}(T)=R_{\mu}(T)\left\{I+\sum_{n=1}^{\infty}(\mu-\lambda)^{n}\left[R_{\mu}(T)\right]^{n}\right.
$$

es un operador en $\mathcal{L}(H)$ y que $\tilde{R}_{\lambda}(T)=R_{\lambda}(T)$.

ii) Para demostrar la fórmula basta hacer notar que

$$
R_{\lambda}(T)-R_{\mu}(T)=R_{\lambda}(T)(\mu I-T) R_{\mu}(T)-R_{\lambda}(T)(\lambda I-T) R_{\mu}(T) .
$$

Claramente la fórmula en (ii) demuestra la conmutatividad de $R_{\lambda}(T)$ y $R_{\mu}(T)$.

Notemos que $R_{\lambda}(T), \lambda \in \rho(T)$, tiene también la siguiente sugestiva representación: 


$$
\begin{aligned}
\mathrm{R}_{\lambda}(T) & =(\lambda I-T)^{-1}=\frac{1}{\lambda-T} \\
& =\frac{1}{\lambda}\left(\frac{1}{1-T / \lambda}\right)=\frac{1}{\lambda}\left(1+\sum_{n=1}^{\infty}\left(\frac{T}{\lambda}\right)^{n}\right)
\end{aligned}
$$

Luego $R_{\lambda}(T)$ existe si $|\lambda|>\|T\|$. Esto demuestra la siguiente proposi ción:

Proposición 4.3. T un operador en $\mathcal{L}(\mathrm{H})$. Entonces $\sigma(\mathrm{T})$ es compacto.

Demostración: Claramente si $\lambda \in \sigma(T)$ entonces $|\lambda| \leq\|T\|$. Luego $\sigma(T)$ es acotado y por lo tanto compacto por ser el complemento del abierto $\rho(T)$.

La siguiente proposición nos dá una propiedad importante de $\sigma(\mathrm{T})$ para un operador autoadjunto.

Proposición 4.4. Sea $T$ un operador en $\mathcal{L}(\mathrm{H})$. Entonces

i) $\quad \sigma\left(T^{*}\right)=\{\lambda: \bar{\lambda} \in \sigma(T)\}$

ii) Si $\mathrm{T}$ es autoadjunto entonces $\sigma(\mathrm{T}) \subseteq \mathbb{R}$ y además

$$
\|T\|=\sup \{|\lambda|: \lambda \in \sigma(T)\}=r(T)
$$

Demostración: La parte (ii) es inmediata de (i). La primera parte es clara del siguiente cálculo. Para $\lambda \in \rho(T)$ se tiene que

$$
R_{\lambda}(T)(\lambda I-T)=I=(\lambda I-T) R_{\lambda}(T)
$$

luego $\left(\bar{\lambda} I-T^{*}\right)\left[R_{\lambda}(T)\right]^{*}=I=R_{\lambda}^{*}(T)\left(\bar{\lambda} I-T^{*}\right)$ y entonces $\bar{\lambda} \in \sigma\left(T^{*}\right)$. La igualdad viene del hecho que $r(T)=\lim _{n \rightarrow \infty}\left\|T^{n}\right\|^{1 / n}$. Para más detalle ver $[1],[3]$. 
Una de las propiedades de la equivalencia unitaria es la siguiente, la cual dejamos como ejercicio.

Proposición 4.5. Sean $T_{1}, T_{2}$ operadores en $\mathcal{L}(\mathrm{H})$. Supongamos que $T_{1}$ sea unitariamente equivalente a $T_{2}$. Entonces $\sigma\left(T_{1}\right)=\sigma\left(T_{2}\right)$.

Nuestro objetivo es formular el teorema espectral para operadores autoadjuntos. Cabe señalar que la teoría se puede generalizar a operadores normales, es decir, operadores que tienen la propiedad de que $T^{*}=T^{*} T$. Veremos que para un operador autoadjunto $T$ es posible encontrar un es pacio de medida $(M, \mu)$ y un operador unitario $U: H \rightarrow \mathcal{L}^{2}(M, \mu)$ tal que

$$
\left(U T U^{-1} f\right)(x)=F(x) f(x)=\left(T_{F} f\right)(x)
$$

para una función $F$ medible y esencialmente acotada a valores reales definida en $M$.

Si $p(x)=\sum_{k=0}^{n} a_{k} x^{k}$ es un polinomio con coeficientes complejos y

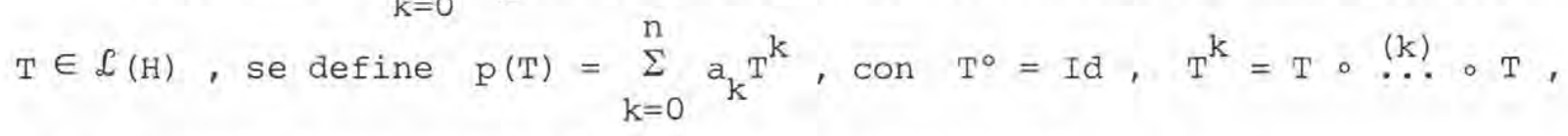
$k \geq 1$, donde la operación "o" significa composición.

Lema 4.6. Sean $\mathrm{p}(\mathrm{x})$ un polinomio $\mathrm{y} \quad \mathrm{T}$ un operador en $\mathcal{L}(\mathrm{H})$. Entonces $\sigma(p(T))=\{p(\lambda): \lambda \in \sigma(T)\}$

Demostración: Sea $\lambda \in \sigma(T)$ y $q(x)=p(x)-p(\lambda)$. Entonces $\lambda$ es una raíz de $q(x)$. Luego $q(x)=(x-\lambda) Q(x)$ y $p(T)-p(\lambda)=(T-\lambda) Q(T)$. Puesto que $T-\lambda$ no tiene inversa entonces $p(T)-p(\lambda)$ no es invertible $y$ por lo tanto $p(\lambda) \in \sigma(p(T))$. 
Supongamos que $\lambda \in \sigma(p(T))$ y $r(x)=p(x)-\lambda$. Consideremos $\mu_{1}, \ldots, \mu_{N}$ las raíces de $r(x)$. Entonces $p(x)-\lambda=a\left(x-\mu_{1}\right) \ldots$ $\ldots\left(x-\mu_{N}\right)$. Si $\mu_{1}, \ldots, \mu_{N} \notin \sigma(T)$ entonces $(p(T)-\lambda)$ es inverti ble lo cual no puede suceder. Por lo tanto para algún i, $\mu_{i} \in \sigma(T)$ y entonces existe $\mu \in \sigma(T)$ tal que $\lambda=p(\mu)$.

Para el caso autoadjunto se puede decir más aún,

Lema 4.7. Sea $T$ un operador autoadjunto de $\mathcal{L}(\mathrm{H})$. Entonces

$$
\|p(T)\|=\sup \{|p(\lambda)|: \lambda \in \sigma(T)\}=\|p\|_{\infty} .
$$

Demostración: Usando parte (vi) del teorema 2.5. y parte (ii) de la pro posición 4.4 . se tiene que:

$$
\begin{aligned}
\|p(T)\|^{2} & =\left\|[p(T)]{ }^{*} p(T)\right\|=\|\overline{p p}(T)\| \\
& =\sup \{|\lambda|: \lambda \in \sigma(p \bar{p}(T))\} \\
& =(\sup \{|p(\lambda)|: \lambda \in \sigma(T)\})^{2} \\
& =\|p\|_{\infty}^{2}
\end{aligned}
$$

Ya estamos en condiciones de definir lo que se denomina "el cálculo funcional contínuo". Mediante este método definiremos $\mathrm{f}(\mathrm{T})$ para una función f contínua. Usaremos primero que los polinomios son densos en $C(\sigma(T))=\{g: \sigma(T) \rightarrow \mathbb{C}$; contínua $\}$ con la norma uniforme \|\|$_{\infty}$ definida por 
$\|g\|_{\infty}=\sup \{g(\lambda): \lambda \in \sigma(T)\} \quad y$ segundo que todo operador acotado sobre un espacio normado $\underline{\bar{x}}$ puede ser extendido a un operador acotado sobre su completación $\underline{\bar{x}}$. Ver [3] para el detalle o cualquier libro clásico de Análisis Funcional.

Teorema 4.8. Sea $T$ un operador autoadjunto en $\mathcal{L}(\mathrm{H})$. Entonces existe una única aplicación $\Phi: \mathrm{C}(\sigma(\mathrm{T})) \rightarrow \mathcal{L}(\mathrm{H})$ con las propiedades siguientes:

a)

$$
\begin{aligned}
& \Phi(f g)=\Phi(f) \Phi(g), \Phi(\lambda f)=\lambda \Phi(f) \\
& \Phi(1)=I, \Phi(\bar{f})=[\Phi(f)]^{*}
\end{aligned}
$$

b) $\Phi$ es isométrico, es decir $\|\Phi(f)\|_{\mathcal{L}(H)}=\|f\|_{\infty}$

c) Sea $f(x)=x$. Entonces $\Phi(f)=T$

d)

$$
\sigma(\Phi(f))=\{f(\lambda): \lambda \in \sigma(T)\}=f(\sigma(T)) .
$$

e)

Si $T x=\lambda x$ entonces $\Phi(f) x=f(\lambda) x$.

Demostración: Por lo ya dicho basta demostrar que:

$$
\|p(T)\|_{\mathcal{L}(H)}=\|p\|_{\infty}
$$

lo cual no es nada más que el lema 4.7. Luego si definimos

$$
\begin{aligned}
& \Phi: P[\sigma(T)] \rightarrow \mathcal{L}(\mathrm{H}) \\
& \Phi(\mathrm{p})=\mathrm{p}(\mathrm{T}), \mathrm{p} \text { polinomio definido en } \sigma(T)
\end{aligned}
$$

obtenemos que se cumplen (a), (b), (c), (d) y (e). La completación de $P[\sigma(T)]$ con la norma \|\|$_{\infty}$ es $C(\sigma(T))$. Luego $\Phi$ se puede extender a $C(\sigma(T))$ preservando todas sus propiedades. 
Ejemplo: Se dice que un operađor $\mathrm{T} \in \mathcal{L}(\mathrm{H})$ es positivo sí y sólo si $\langle T f, f\rangle \geq 0$ para todo $f \in H$. No es difícil demostrar que $\sigma(T) \subseteq[0, \infty]$. Entonces la función $f(x)=\sqrt{x}$ pertenece a $C(\sigma(T))$. Esto demuestra la existencia de $\sqrt{\mathrm{A}}$ para un operador positivo.

$\S 5$. Medidas espectrales.

Antes de entrar en detalles necesitamos el siguiente teorema que caracteriza los funcionales lineales positivos de $c(\overline{\bar{x}})$, donde $C(\underline{\bar{x}})=\{f: \underline{\bar{x}} \rightarrow \mathbb{C} ; f$ contínua , $\bar{x}$ compacto $\}$.

Definición 5.1. Un funcional $\ell \in[C(\underline{\bar{X}})]^{*}$ se dice positivo si $\ell(f) \geq 0$ para todo $f \geq 0$.

Recordemos que un subconjunto $A$ de un espacio topológico $\underline{\bar{x}}$ es un $G_{f}$ sí y sólo si es la intersección numerable de abiertos de $\bar{x}$.

Teorema 5.2. (Reiz-Markov). Sea $\underline{\bar{x}}$ un espacio compacto y Hausdorff. En tonces para todo funcional positivo $\ell \in[\mathrm{C}(\underline{\bar{X}})]^{*}$ existe una medida $\mu$ definida en la $\sigma$-álgebra generada por los $G_{\delta}$ en el compacto $\underline{\bar{x}}$ tal que

$$
\ell(\mathrm{f})=\int_{\frac{\bar{x}}{}} \mathrm{fd} \mu .
$$

Para la demostración de este teorema ver [4].

$$
\text { Para } T \text { autoadjunto definamos en } C(\sigma(T)) \text { el siguiente funcional }
$$




$$
\ell(f)=\langle\varphi, f(T) \varphi\rangle .
$$

De ahora en adelante para evitar problemas de notación supondremos que el producto interno es $\mathbb{C}$-lineal en la segunda componente y conjugado lineal en la primera.

Es decir

$$
\left\langle\alpha \varphi_{1}, \beta \varphi_{2}\right\rangle=\overline{\alpha \beta}\left\langle\varphi_{1}, \varphi_{2}\right\rangle \quad \alpha, \beta \in \mathbb{C}, \quad \varphi_{1}, \varphi_{2} \in \mathrm{H} .
$$

En esta nueva convención $l$ es un funcional lineal acotado. Además pa$r a f \geq 0, f \in C(\sigma(T))$ existe $g \in C(\sigma(T))$ tal que $g \cdot g=f$. Entonces para $\varphi \in \mathrm{H}$ tenemos que (T autoadjunto)

$$
\begin{aligned}
\ell(f) & =\langle\varphi, f(T) \varphi\rangle=\langle\varphi,(g \cdot g)(T) \varphi\rangle \\
& =\langle\varphi, g(T) g(T) \varphi\rangle=\langle g(T) \varphi, g(T) \varphi\rangle \\
& =\|g(T) \varphi\|^{2} \geq 0
\end{aligned}
$$

lo cual demuestra que $l$ es un funcional positivo.

$$
\text { Aplicando el teorema 5.2. obtenemos que existe una medida } \mu_{\varphi}
$$

tal que

$$
\langle\varphi, f(T) \varphi\rangle=\int_{\sigma(T)} f(\lambda) d \mu .
$$

Usando la notación del teorema 4.8. la igualdad se escribe también Como:

$$
\langle\varphi, \Phi(f) \varphi\rangle=\int_{\sigma(T)} f(\lambda) d \mu_{\varphi} .
$$


Definición 5.3. A la medida $\mu_{\varphi}, \varphi \in \mathrm{H}$, la llamaremos medida espectral asociada a $\varphi$.

Definición 5.4. Un vector $\boldsymbol{\varphi} \in \mathrm{H}$ es llamado cíclico para $\mathrm{T}$ si el conjunto $\mathrm{H}_{1}=\{\mathrm{p}(\mathrm{T}) \varphi: \mathrm{p}$ un polinomio $\}$ es denso en $\mathrm{H}$.

Para los operadores autoadjuntos con vector cíclico se tiene el siguiente teorema espectral

Teorema 5.5. Sea $T$ un operador autoadjunto con vector cíclico $\varphi$. Enton ces existe un operador unitario $U, U: H \rightarrow \mathcal{L}^{2}(\sigma(T), \mu)$ tal que

$$
\left(U U^{-1} f\right)(\lambda)=\lambda f(\lambda) .
$$

Demostración: Definamos $\mathrm{H}_{1}=\{\Phi(\mathrm{f}) \varphi: \mathrm{f} \in \mathrm{C}(\sigma(\mathrm{T}))\}$. Aplicaremos un argumento de extensión similar al usado en la demostración del teorema 4.8. Definimos $\mathrm{U}: \mathrm{H}_{1} \rightarrow \mathrm{C}(\sigma(\mathrm{T}))$ como

$$
U(\Phi(f) \varphi)=f
$$

Las propiedades de $\Phi$ y la definición de $\mu_{\psi}$ justifican los siguientes cálculos

$$
\begin{aligned}
& \langle U \Phi(f) \varphi, U \Phi(g) \varphi\rangle_{L^{2}}=\langle f, g\rangle{ }_{L^{2}} \\
& \langle\Phi(f) \varphi, \Phi(g) \varphi\rangle_{H}=\left\langle\varphi, \Phi^{*}(f) \Phi(g) \varphi\right\rangle_{H} \\
& =\langle\varphi, \Phi(\bar{f}) \Phi(g) \varphi\rangle_{H} \\
& =\langle\psi, \Phi(\bar{f} g) \varphi\rangle_{H}
\end{aligned}
$$




$$
=\int_{\sigma(T)} \overline{f g d \mu_{\varphi}}=\langle f, g\rangle_{\mathcal{L}^{2}}
$$

los cuales demuestran que $U$ es unitario. Lo único que nos falta justificar es que $U$ está bien definido, es decir, si $f=g$ en $\mathcal{L}^{2}\left(\sigma(T), \mu_{\varphi}\right)$ entonces $\Phi(f) \varphi=\Phi(g) \varphi$. Esto se ve claro si tomamos $g=f$ en el cálculo anterior obteniendo que:

$$
\|\Phi(f)\|^{2}=\int_{\sigma(T)}|f|^{2} d \mu_{\varphi} .
$$

Luego si $h=0$ casi en todas partes entonces $\|\Phi(h) \varphi\|=0$ y así $\|\Phi(f) \varphi-\Phi(g) \psi\|=\|\Phi(f-g) \psi\|=0$ lo cual prueba que $\mathrm{U}$ está bien definida. Finalizamos la construcción de U usando el argumento de extensión ya mencionado para $\mathrm{H}_{1}$, el cual es denso en $\mathrm{H}$, y también aplicando la densidad de $C(\sigma(T))$ en $\mathcal{L}^{2}\left(\sigma(T), \mu_{\varphi}\right)$.

En resumen hemos definido un operador unitario $U$ de $H$ en $\mathcal{L}^{2}\left(\sigma(T), \mu_{\psi}\right)$. Aún más, hemos demostrado que $\mathrm{H}$ y $\mathcal{L}^{2}\left(\sigma(T), \mu_{\psi}\right)$ son espacios de Hilbert isomorfos. Finalizamos la demostración probando la fórmula dada en el teorema. Por densidad es suficientedemostrarla para funciones contínuas. Para mayor claridad de la demostración llamaremos g a la función en $C(\sigma(T))$ definida por $g(x)=x$. Para $f \in C(\sigma(T))$ se tiene que

$$
\begin{aligned}
\left(\mathrm{UTU}^{-1}\right)(f)(\lambda) & =(\mathrm{UT} \Phi(f) \varphi)(\lambda) \\
& =(U \Phi(g) \Phi(f) \psi)(\lambda)
\end{aligned}
$$




$$
\begin{aligned}
& =(U \Phi(g f) \psi)(\lambda) \\
& =g(\lambda) f(\lambda)=\lambda f(\lambda) .
\end{aligned}
$$

La segunda igualdad viene del teorema 4.8. parte (a) y las dos últimas de las propiedades de $\Phi$ y la definición de U respectivamente.

Ahora ya estamos en condiciones para dar el teorema espectral para un operador $\mathrm{T}$ autoadjunto cualquiera.

Teorema 5.6. Sea $T$ autoadjunto en $\mathcal{L}(\mathrm{H}), \mathrm{H}$ espacio de Hilbert separable. Entonces existe una sucesión $\left\{\mu_{n}\right\}_{n=1}^{N}, N=1,2, \ldots$ de medidas con soporte en $\sigma(\mathrm{T})$ y un operador unitario $\mathrm{U}$

$$
\mathrm{U}: \mathrm{H} \rightarrow \underset{\mathrm{n}=1}{\mathrm{~N}} \mathcal{L}^{2}\left(\mathbb{R}, \mu_{\mathrm{n}}\right)
$$

tal que

$$
\left(\mathrm{UTU}^{-1} \mathrm{f}\right)_{\mathrm{n}}(\lambda)=\lambda_{\mathrm{f}}(\lambda)
$$

donde $\mathrm{f} \in \underset{\mathrm{n}=1}{\oplus} \mathcal{L}^{2}(\mathbb{R}, \mu)$ lo escribimos como la $\mathrm{N}$-tupla $f=\left(\mathrm{f}_{\mathrm{n}}\right)_{\mathrm{n}=1}^{\mathrm{N}}$.

Demostración: No es difícil demostrar que podemos encontrar una sucesión de vectores $\left\{\varphi_{n}\right\}_{n=1}^{N}, N=1,2, \ldots$ ó $\infty$ tal que $H=\underset{n=1}{\oplus} H_{n}$ donde $\mathrm{H}_{\mathrm{n}}$ es la clausura de $\left\{\mathrm{f}(\mathrm{T}) \varphi_{\mathrm{n}}: \mathrm{f} \in \mathrm{C}(\sigma(\mathrm{T}))\right\}$ y $\mathrm{T}$ deja invariante cada $H_{n}$. Cada $H_{n}$ es un espacio de Hilbert y $T$ restringido a cada $\mathrm{H}_{\mathrm{n}}$ tiene un vector cíclico $\varphi_{\mathrm{n}}$. Luego para cada $\mathrm{n}$ podemos aplicar el teorema 5.5. obteniendo $U_{n}$ tal que 


$$
\left(U_{n} T U_{n}^{-1} f\right)_{n}(\lambda)=\lambda f_{n}(\lambda)
$$

Luego si definimos $U=\left(U_{n}\right)_{n=1}^{N}$ vemos que $U$ es unitario y satisface la ecuación requerida.

$$
\text { Hacemos notar que } \mu_{n}(\mathbb{R})=\left\|\varphi_{n}\right\| \text {. Si consideramos } M=\underset{n=1}{U} \mathbb{R},
$$
$\mathrm{N}$-copias de $\mathbb{R}$, definimos una medida $\mu$ finita en $M$ de la siguiente manera: $\left.\mu\right|_{\mathbb{R}_{n}}=\mu_{n}$. Luego

$$
\mu(M)=\sum_{n=1}^{N} \mu_{n}(\mathbb{R})=\sum_{n=1}^{N}\left\|\varphi_{n}\right\|
$$

Eligiendo los $\varphi_{n}$ de tal manera que $\left\|\Psi_{n}\right\|=2^{-n}$ se tiene que $\mu(M)=1$. Entonces podemos construir un espacio de medida finita $(M, \mu)$ tal que $\mathrm{U}: \mathrm{H} \rightarrow \mathcal{L}^{2}(\mathrm{M}, \mu)$ tiene la propiedad de que

$$
\left(U T U^{-1} \mathrm{f}\right)(\mathrm{m})=\mathrm{F}(\mathrm{m}) \mathrm{f}(\mathrm{m})
$$

con $F$ una función acotada en $M$.

Sabemos que toda medida $\mu$ en $\mathbb{R}$ puede descomponerse en su parte absolutamente contínua respecto a la medida de Lebesgue, su parte singular y su componente discreta. En otras palabras:

$$
\mu=\mu_{a c}+\mu_{s}+\mu_{d}
$$

Esta descomposición de $\mu$ hace que $\mathcal{L}^{2}(\mathbb{R}, \mu)$ se descomponga a su vez en 


$$
\mathcal{L}^{2}(\mathbb{R}, \mu)=\mathcal{L}^{2}\left(\mathbb{R}, \mu_{\mathrm{ac}}\right) \oplus \mathcal{L}^{2}\left(\mathbb{R}, \mu_{\mathrm{s}}\right) \oplus \mathcal{L}^{2}\left(\mathbb{R}, \mu_{\mathrm{d}}\right) .
$$

Definición 5.7. Sea $T$ un operador autoadjunto en $\mathcal{L}(\mathrm{H})$. Definimos

$$
\begin{aligned}
& \mathrm{H}_{\mathrm{ac}}=\left\{\varphi: \mu_{\varphi} \text { es absolutamente contínua }\right\} \\
& \mathrm{H}_{\mathrm{s}}=\left\{\varphi: \mu_{\varphi} \text { es singular }\right\} \\
& \mathrm{H}_{\mathrm{d}}=\left\{\varphi: \mu_{\varphi} \text { es discreta }\right\}
\end{aligned}
$$

Todas las descomposiciones son respecto a la medida de Lebesgue.

Teorema 5.8. Sea $\mathrm{T}$ un operador autoadjunto en $\mathcal{L}(\mathrm{H})$. Entonces

i)

$$
\mathrm{H}=\mathrm{H}_{\mathrm{ac}} \oplus \mathrm{H}_{\mathrm{s}} \oplus \mathrm{H}_{\mathrm{d}}
$$

ii) Cada uno de estos subespacios son invariantes bajo $\mathrm{T}$.

ii) $\left.\quad \mathrm{T}\right|_{\mathrm{ac}}$ tiene solamente medidas espectrales absolutamente contínuas, $\left.T\right|_{H_{S}}$ tiene solamente medidas espectrales singulares y $\left.\mathrm{T}\right|_{\mathrm{H}_{\mathrm{d}}}$ tiene un conjunto completo de vectores propios.

La demostración la dejamos de ejercicio y es una bonita aplicación de el análisis clásico al análisis funcional.

Ejemplos: a) Consideremos el caso finito dimensional. Sea $T$ una matriz de $n \times n$ autoadjunta. Se sabe de álgebra lineal que tales matrices tienen $\psi_{1}, \ldots, \psi_{n}$ vectores propios que forman una base de $\mathbb{C}^{\mathrm{n}}$. Es decir $A \psi_{i}=\lambda_{i} \psi_{i}, \quad i=1,2, \ldots, n$. Supongamos que todos los valores propios 
$\lambda_{i}$ son diferentes. Consideramos las medidas de Dirac $\mu_{i}=\delta\left(x-\lambda_{i}\right)$, es decir $\delta\left(x-\lambda_{i}\right)(f)=f\left(\lambda_{i}\right)$ donde $f$ es una función.

$$
\begin{gathered}
\text { Definamos } \mu=\sum_{i=1}^{n} \mu_{n} . \text { El operador } s \text { definido por } \\
s: \mathcal{L}^{2}(\mathbb{R}, \mu) \rightarrow \mathbb{C} \\
s(f)=\left(f\left(\lambda_{1}\right), \ldots, f\left(\lambda_{n}\right)\right)
\end{gathered}
$$

es un operador unitario puesto que

$$
\begin{aligned}
\langle s f, s g\rangle_{\mathbb{C}^{n}} & =\sum_{i=1}^{n} f\left(\lambda_{i}\right) g \overline{\left(\lambda_{i}\right)} \\
& =\int_{\mathbb{R}} f \bar{g} d_{\mu} \\
& =\langle f, g\rangle_{\mathcal{L}^{2}(\mathbb{R}, \mu)}
\end{aligned}
$$

Por 10 tanto hemos demostrado que $\mathscr{L}^{2}(\mathbb{R}, \mu)$ es isomorfo a $\mathbb{C}^{\mathrm{n}}$.

Es fácil demostrar que (TS) (f) $=S(g f)$ donde $g(\lambda)=\lambda$, lo cual prueba que $S^{-1} \operatorname{TS}(f)(\lambda)=\lambda f(\lambda)$ y por 10 tanto $T$ es unitariamente equivalente al operador multiplicación por $\lambda$ en $\mathcal{L}^{2}(\mathbb{R}, \mu)$. Investigue qué sucede si T tiene valores propios con multiplicidad mayor que uno.

Ejemplo: Sea $H=\ell^{2}(\mathbb{R})=\left\{\left\{a_{n}\right\}_{n=-\infty}^{+\infty} \subseteq \mathbb{R}: \sum_{n=-\infty}^{+\infty}\left|a_{n}\right|^{2}<\infty\right\}$. Sea $T$ el operador corrimiento definido en $\ell^{2}(\mathbb{R})$ por $(\mathrm{Ta})_{\mathrm{n}}=\mathrm{a}_{\mathrm{n}+1}$. Es fácil demostrar que $T^{*}$ está dado por $\left(T^{*} a\right)_{n}=a_{n-1}$. Definamos $A=T+T^{*}$. 
obviamente $T$ es autoadjunto y si definimos el operador unitario $\mathrm{U}: \mathrm{H} \rightarrow \mathcal{L}^{2}([0,1])$ dado por

$$
U\left(\left\{a_{n}\right\}_{n=-\infty}^{+\infty}\right)=\sum_{n=-\infty}^{+\infty} a_{n} e^{2 \pi i n x}
$$

obtenemos que $\mathrm{UAU}^{-1}$ es el operador multiplicación por $2 \cos (2 \pi \mathrm{x})$ en $\mathcal{L}^{2}([0,1])$.

Finalmente terminamos este Capítulo haciendo la observación de que el teorema espectral es también válido para operadores normales, $\mathrm{T}^{*} \mathrm{~T}=\mathrm{TT}^{*}$. Tal generalización será necesaria para extender el teorema espectral a operadores no acotados. 
CAPITULO II. OPERADORES NO ACOTADOS.

En la práctica una gran mayoría de los operadores en física matemática resultan ser no acotados como por ejemplo el operador posición, el operador energía, etc. Nos van a interesar algunos operadores lineales T que son densamente definidos, es decir, su dominio $D(T)$ es un subespa cio denso del espacio de Hilbert H . Para motivar las definiciones y teoremas de este Capítulo, daremos algunos ejemplos de tales operadores.

Ejemplo 1. (Operador posición). Sea $\mathrm{H}$ el espacio de Hilbert $\mathcal{L}^{2}$ (IR) $\mathrm{y}$ $D(T)$ el subespacio definido por $D(T)=\left\{f \in \mathcal{L}^{2}(\mathbb{R}): \int x^{2}|f(x)|^{2} d x<\infty\right\}$. El operador definido en $D(T)$ por

$$
\mathrm{T}: \mathrm{D}(\mathrm{T}) \rightarrow \mathrm{H},(\mathrm{Tf})(\mathrm{x})=\mathrm{xf}(\mathrm{x})
$$

lo llamaremos el operador posición.

Es claro de la definición de $D(T)$ que $T f \in H, \forall f \in D(T)$, luego $T$ está bien definido. Definamos para cada $N \in \mathbb{N}$ la sucesión de función en H dada por

$$
f_{N}(x)=\left\{\begin{array}{lll}
\frac{1}{\sqrt{N}} & \text { si } & x \in[0, N] \\
0 & \text { si } & x \notin[0, N]
\end{array}\right.
$$


Obviamente $\left\|f_{\mathrm{N}}\right\|_{\mathcal{L}^{2}(\mathbb{R})}=1$ y además

$$
\|\mathrm{T}\|=\sup \left\{\|\mathrm{Tf}\|_{\mathcal{L}^{2}(\mathbb{R})}:\|\mathrm{f}\|=1\right\} \geq\left\|\mathrm{Tf} \mathrm{N}_{\mathrm{N}}\right\|=\sqrt{\frac{1}{3}} \cdot \mathrm{N}
$$

lo cual demuestra que $T$ no es acotada.

Ejemplo 2. Sea $H=\mathscr{L}^{2}(\mathbb{R})$ y $D(T)=S(\mathbb{R})$ el espacio de las funciones tales que ella y sus derivadas convergen a cero cuando $n$ tiende a infinito más rapidamente que cualquier polinomio, es decir, f $\in S(\mathbb{R})$ sí y sólo si f es infinitamente diferenciable y

$$
\sup _{x \in \mathbb{R}}\left|x^{\alpha}{ }^{\beta} f(x)\right|<\infty .
$$

Definamos $\quad \mathrm{T}: \mathrm{D}(\mathrm{T}) \rightarrow \mathrm{H}$ por

$$
(T f)(x)=-\frac{d^{2} f(x)}{d x^{2}}+x^{2} f(x) \text {. }
$$

Claramente $\mathrm{T}$ está bien definido y es lineal puesto que si $\mathrm{f} \in \mathrm{S}(\mathbb{R})$ entonces $\mathrm{T} f \in S(\mathbb{R}) \subseteq \mathcal{L}^{2}(\mathbb{R})$. Denotemos por $\varphi_{\mathrm{n}}$ las funciones de Hermite dadas por

$$
\varphi_{n}(x)=\left(2^{n} n !\right)^{-1 / 2}(-1)^{n} \pi^{-1 / 4} \exp \left(\frac{1}{2} x^{2}\right) \frac{d^{n}}{d x^{n}}\left(e^{-x^{2}}\right)
$$

No es difícil demostrar que $\left\|\varphi_{n}\right\|=1$ y que

$$
T \varphi_{n}=(2 n+1) \varphi_{n}
$$


Por lo tanto $T$ no es acotado puesto que

$$
\begin{aligned}
\|T\| \geq\left\|T \varphi_{n}\right\|_{\mathcal{L}^{2}(\mathbb{R})}^{2} & =\int(2 n+1)^{2} \varphi_{n}^{2}(x) d x=(2 n+1)^{2}\left\|\varphi_{n}\right\| \mathcal{L}^{2}(\mathbb{R}) \\
& =(2 n+1)^{2} .
\end{aligned}
$$

En mecánica cuántica existen operadores T (como por ejemplo el operador posición) que no son acotadas y que satisfacen en algún sentido

$$
\langle\mathrm{Tf}, \mathrm{g}\rangle=\langle\mathrm{f}, \mathrm{Tg}\rangle .
$$

Veremos en la próxima sección que los operadores no acotados no pueden estar definidos en todo H.

\section{§ 1. Operadores cerrados y adjuntos.}

Definición 1.1. El gráfico $\Gamma(T)$ de un operador lineal (no necesariamente acotado) es el subconjunto del espacio de Hilbert $\mathrm{H} \times \mathrm{H}$ definido por $\Gamma(T)=\{(f, T f): f \in D(T)\}$. Diremos que el operador $T$ es cerrado sí y sólo si $\Gamma(\mathrm{T})$ es un subconjunto cerrado de $\mathrm{H} \times \mathrm{H}$.

Definición 1.2. Sean $T_{1} \quad Y \quad T$ operadores lineales con dominios $D\left(T_{1}\right)$ y $D(T)$ respectivamente, ambos densamente definidos en un espacio de Hilbert $H$. Si $\Gamma\left(T_{1}\right) \supset \Gamma(T)$ entonces diremos que $T_{1}$ es una extensión de $\mathrm{T} y$ denotaremos este hecho por $\mathrm{T}_{1} \supset \mathrm{T}$. Equivalentemente $\mathrm{T}_{1} \supset \mathrm{T}$ sí y sólo si $\mathrm{D}\left(\mathrm{T}_{1}\right) \supset \mathrm{D}(\mathrm{T})$ y $\mathrm{T}_{1} \mathrm{f}=\mathrm{Tf}$ para todo $\mathrm{f}$ en $\mathrm{D}(\mathrm{T})$. 
Definición 1.3. Diremos que $T$ es clausurable si posee una extensión que es cerrada. Cualquier operador clausurable posee una extensión cerrada minimal. A esta extensión más pequeña la llamaremos la clausura de T y la denotaremos por $\overline{\mathrm{T}}$.

Es bien conocido del análisis funcional el teorema siguiente [3]:

Teorema 1.4. (Teorema del gráfico cerrado). Sean $\underline{\bar{X}}$ e $\underline{\bar{Y}}$ espacios de Banach y $T: \underline{\bar{x}} \rightarrow \underline{\bar{y}}$ un operador lineal. Entonces $T$ es acotado sí y sólo si $\Gamma(T)$ es cerrado en $\underline{\bar{X}} \times \underline{\bar{Y}}$.

Corolario 1.5. (Hellinger-Toeplitz). Sea $\mathrm{T}: \mathrm{H} \rightarrow \mathrm{H}$ operador lineal definido en $H$, es decir, $D(T)=H$. Supongamos $\langle\mathrm{Tf}, \mathrm{g}\rangle=\langle\mathrm{f}, \mathrm{Tg}\rangle$ para todo $f y$ en $H$. Entonces $T$ es acotado.

Demostración: Por teorema 1.4. basta probar que $\Gamma(T)$ es cerrado. Sea $\left(f_{n}, T f_{n}\right)$ una sucesión en $\Gamma(T)$ que converge a (f,g). Necesitamos de mostrar que $(f, g)$ pertenece a $\Gamma(T)$, es decir, $T f=g$. Pero para todo $h$ en $H$ se tiene que

$$
\begin{aligned}
\langle h, g\rangle & =\lim _{n \rightarrow \infty}\left\langle h, T f_{n}\right\rangle=\lim _{n \rightarrow \infty}\left\langle T h, f_{n}\right\rangle \\
& =\langle T h, f\rangle=\langle h, T f\rangle
\end{aligned}
$$

luego

$$
\langle h, g\rangle=\langle h, T f\rangle, \quad \forall h \in H .
$$


Por lo tanto $\mathrm{Tf}=\mathrm{g}$. Lo cual finaliza la demostración.

Este Corolario nos dice que los operadores no acotados que cumplen la conđición

$$
\langle\mathrm{Tf}, \mathrm{g}\rangle=\langle\mathrm{f}, \mathrm{Tg}\rangle
$$

no pueden estar definidos en todo H . Este es el motivo porque los operadores no acotados están definidos solamente en un subespacio propio de H . La siguiente proposición nos da información acerca del gráfico de $\bar{T}$ y nos ilustra una técnica muy usada en la teoría de operadores no acotados.

Proposición 1.6. Si $T: D(T) \rightarrow H$ es clausurable entonces $\Gamma(\bar{T})=\overline{\Gamma(T)}$. Demostración: Supongamos que $S$ es una extensión cerrada de T . Claramente $\overline{\Gamma(T)} \subset \overline{\Gamma(S)}=\Gamma(S)$. Además si $(0, \phi) \in \overline{\Gamma(T)}$ entonces $0=\mathrm{S}(0)=\phi$ y como $\mathrm{S}(0)=\mathrm{T}(0)$ se tiene que $\phi=0$. Definamos $\mathrm{R}$ el operador lineal con dominio $D(R)=\{\phi:(\phi, \varphi) \in \overline{\Gamma(T)}$, para algún $\varphi\}$ y $R(\phi)=\varphi$. Por definición de $R$ se tiene que $\Gamma(R)=\overline{\Gamma(T)}$ y por lo tanto $\Gamma(R)$ es una extensión cerrada de $\Gamma(T)$ con la propiedad de que $\Gamma(\mathrm{R}) \subset \Gamma(\mathrm{S})$. Como $\mathrm{S}$ era una extensión cerrada arbitraria de $\mathrm{T}$ se tiene que $R=\bar{T}$.

Ahora extenderemos la noción de operador adjunto al caso no aco tado. Notemos que para definir un operador no acotado $T$ debemos espe cificar su dominio D(T) y como actúa en los elementos de $D(T)$. En particular dos operadores no acotados son iguales si sus dominios coin- 
ciden y sus valores en un mismo elemento del dominio son los mismos. Para definir el operador adjunto necesitamos entonces especificar su dominio y como actúa en tales elementos.

Definición 1.7. Sea $T: D(T) \rightarrow H$ un operador lineal densamente definido. Definimos $D\left(T^{*}\right)$ como sigue

$$
\mathrm{D}\left(\mathrm{T}^{*}\right)=\{\phi \in \mathrm{H}: \text { existe } \varphi \in \mathrm{H} \text { tal que }\langle\mathrm{T} \psi, \phi\rangle=\langle\psi, \varphi\rangle, \forall \psi \in \mathrm{D}(\mathrm{T})\}
$$

Para $\phi \in D\left(T^{*}\right)$ definimos $T^{*} \phi=\varphi$. $T^{*}$ es llamado el autoadjunto de T.

Afirmamos que $T^{*}$ está bien definido. Supongamos que $T^{*} \varphi=\phi_{1}$ y $T^{*} \varphi=\phi_{2}$ para algún $\varphi \in D\left(T^{*}\right)$. Entonces $\left\langle\psi, \phi_{1}\right\rangle=\left\langle\psi, \phi_{2}\right\rangle$ para todo $\psi \in \mathrm{D}(\mathrm{T})$. Como $\mathrm{D}(\mathrm{T})$ es denso en $\mathrm{H}$ se obtiene que $\phi_{1}=\phi_{2}$. Esto nos dice que $T^{*}$ está definido sí y sólo si $\mathrm{D}(\mathrm{T})$ es denso en $\mathrm{H}$.

Notemos que $D\left(T^{*}\right)$ es un subespacio que no es necesariamente denso en $H$. Aún más se pueden construir ejemplos en que $D\left(T^{*}\right)=\{0\}$.

Teorema 1.8. Sean $T$ y $S$ operadores lineales densamente definidos en H. Entonces

i) $\quad \mathrm{Si} \mathrm{S} \subset \mathrm{T}$ entonces $\mathrm{T}^{*} \subset \mathrm{S}^{*}$

ii) $\quad T^{*}$ es cerrado

ii) $\quad T$ es clausurable sí y sólo si $D\left(T^{*}\right)$ es denso y entonces $\bar{T}=\left(T^{*}\right)^{*}$.

iv) Si $\mathrm{T}$ es clausurable entonces $(\overline{\mathrm{T}})^{*}=\mathrm{T}^{*}$ 
Demostración: i) Es obvio de las definiciones.

ii) Definamos el operador unitario $\mathrm{V}$ en $\mathrm{H} \times \mathrm{H}$ dado por $\mathrm{V}(\mathrm{f}, \mathrm{g})=$ $=(-g, f)$. Todo operador unitario tiene la propiedad de que $\mathrm{V}\left(\mathrm{E}^{\perp}\right)=$ $=(\mathrm{V}(\mathrm{E}))^{\perp}$. El complemento ortogonal es siempre un subespacio cerrado. Por lo tanto basta demostrar que $\Gamma\left(T^{*}\right)=V\left([\Gamma(T)]^{\perp}\right.$ ) lo cual es fácil ver.

iii) En una dirección es inmediato puesto que

$$
\overline{\Gamma(T)}=\left([\Gamma(T)]^{\perp}\right)^{\perp}=\left(V^{2}\left(\Gamma(T)^{\perp}\right)^{\perp}=\left(V\left(\Gamma\left(T^{*}\right)\right)^{\perp}\right.\right.
$$

Para el converso supongamos que $D\left(T^{*}\right)$ no es denso en $H$. Luego existe $\psi \in\left[D\left(T^{*}\right)\right]^{\perp}$. Se ve fácilmente que $(\psi, 0)$ pertenece a $\left[\Gamma\left(T^{*}\right)\right]^{\perp}$ por lo tanto $V\left(\Gamma\left(T^{*}\right)\right)^{\perp}$ no es el gráfico de un operador. Entonces $T$ no es clausurable puesto que $\overline{\Gamma(T)}=\left(\mathrm{V}\left(\Gamma\left(\mathrm{T}^{*}\right)\right)^{\perp}\right.$.

iv) Es obvio puesto que $\mathrm{T}^{*}=\overline{\mathrm{T}^{*}}=\mathrm{T}^{* * *}=(\overline{\mathrm{T}})^{*}$

Definición 1.9. Sea $T$ un operador densamente definido en $H$. Diremos que $\mathrm{T}$ es simétrico si $\mathrm{T} \subset \mathrm{T}^{*}$, es decir, $\mathrm{si} \mathrm{D}(\mathrm{T}) \subset \mathrm{D}\left(\mathrm{T}^{*}\right.$ ) (luego $D\left(T^{*}\right)$ es denso en $\left.H\right)$ y $T \phi=T^{*} \phi$ para todo $\phi$ en $D(T)$. Equivalen temente $T$ es simétrico sí y sólo si

$$
\langle T \phi, \psi\rangle=\langle\phi, T \psi\rangle
$$

para todo $\phi, \psi$ en $D(T)$.

Definición 1.10. $\mathrm{T}$ es autoadjunto si $\mathrm{T}=\mathrm{T}^{*}$, esto es, sî y sólo si $\mathrm{T}$ es simétrico y $D(T)=D\left(T^{*}\right)$. 
Un operador simétrico es siempre clausurable puesto que $D(T) \subset D\left(T^{*}\right)$ y $D(T)$ es denso en $H$. De estas definiciones es fácil probar la siguiente proposición

Proposición 1.11. i) $\mathrm{Si} T$ es simétrico entonces $\mathrm{T} \subset \mathrm{T}^{\star *} \subset \mathrm{T}^{*}$

ii) Si $\mathrm{T}$ es simétrico y cerrado entonces $\mathrm{T}=\mathrm{T}^{* *} \subset \mathrm{T}^{*}$

iii) Si $\mathrm{T}$ es autoadjunto $\mathrm{T}=\mathrm{T}^{* *}=\mathrm{T}^{*}$.

Ahora daremos un criterio para saber cuando un operador simétrico es autoadjunto.

Teorema 1.12. Sea $T$ un operador simétrico en $\mathrm{H}$. Entonces son equiva lentes
a) $T$ es autoadjunto
b) $\quad T$ es cerrado $y \operatorname{Ker}\left(T^{*} \pm i\right)=\{0\}$
c) $\quad \operatorname{Ran}(\mathrm{T} \pm \mathrm{i})=\mathrm{H}$.

Demostración: a) $\Rightarrow$ b). Si $T$ es autoadjunto entonces $D(T)=D\left(T^{*}\right)$ por lo tanto si existe $\phi$ en $D\left(T^{*}\right)$ tal que $T^{*} \phi=i \phi$ se tiene que (recordamos que estamos con el producto interno que es conjugado en la primera variable)

$$
-i\langle\phi, \phi\rangle=\langle i \phi, \phi\rangle=\langle T \phi, \phi\rangle=\langle\phi, T \phi\rangle=i\langle\phi, \phi\rangle
$$

y esto puede suceder solamente si $\phi=0$. 
b) $\Rightarrow c)$. Probaremos que Ran $(T-I)$ es denso en H. E1 argumento para $\operatorname{Ran}(T+i)$ es similar. Si $\operatorname{Ran}(T-i)$ no fuera denso en $H$ se tiene que existe $\phi \neq 0$ tal que $\phi$ pertenece a $[\operatorname{Ran}(T-i)]^{\perp}$. Entonces $\langle(T-i) \psi, \phi\rangle=0$ para todo $\psi$ en $D(T)$. Luego $\phi \in D\left(T^{*}\right) \quad y$ $(T-i){ }^{*} \phi=(T+i) \phi=0$ lo cual contradice (b). Por 10 tanto basta de mostrar que Ran $(T-i)$ es cerrado. Sea $H=(T-i I)$. Consideremos $\left\{\phi_{n}\right\}$ una sucesión en $D(T)$ tal que $H \phi_{n}$ converge a $\psi$. Debemos demostrar que existe $\psi_{0}$ en $D(T)$ tal que $H \psi_{0}=\psi$.

La igualdad siguiente que es vâlida para todo $n$ en $D(T)$

$$
\|H n\|^{2}=\|\mathrm{Tn}\|^{2}+\|n\|^{2}
$$

muestra que $T \phi_{\mathrm{n}} \mathrm{y} \phi_{\mathrm{n}}$ son sucesiones de Cauchy en $\mathrm{H}$, por lo tanto existe $\psi_{0}$ tal que $\phi_{\mathrm{n}}$ converge a $\psi_{0}$. Como $\mathrm{T}$ es cerrado se tiene que $T \phi_{\mathrm{n}}$ converge a $\mathrm{T} \psi_{\text {。 }}$ y por unicidad del límite se obtiene que $\mathrm{H} \psi_{\mathrm{o}}=\psi$.

c) $\Rightarrow$ a). Sea $\phi \in D\left(T^{*}\right)$. Como $\operatorname{Ran}(T-i)=H$ existe $\eta$ en $D(T)$ tal que $(T-i) \eta=\left(T^{*}-i\right) \phi$. Pero $D(T) \subset D\left(T^{*}\right)$ luego $\phi-n \in D\left(T^{*}\right) \quad y$ $\left(T^{*}-i\right)(\phi-\eta)=0$. Se sabe que $\operatorname{Ker}\left(T^{*}-i\right)=[\operatorname{Ran}(T+i)]^{\perp}=H^{\perp}=\{0\}$. Por 10 tanto $\phi-\eta=0$ y entonces $\phi \in D(T)$ y $D\left(T^{*}\right)=D(T)$.

Ejemplo: Sea $T$ el operador posición en $\mathcal{L}^{2}(\mathbb{R})$, es decir,

$$
(\mathrm{Tf})(\mathrm{x})=\mathrm{xf}(\mathrm{x})
$$

con dominio $\mathrm{D}(\mathrm{T})$ dado por 


$$
D(T)=\left\{f \in \mathscr{L}^{2}(\mathbb{R}): \int_{\mathbb{R}} x^{2}|f(x)|^{2} d x<\infty\right\}
$$

Como ya vimos anteriormente $T$ es un operador no acotado. Es fácil ver que $T$ es simétrico. Demostraremos vía teorema 1.12. que $T$ es cerrado. Sea $f \in \mathcal{L}^{2}(\mathbb{R})$ entonces $g=f h$ con $h(x)=(x+i)^{-1}$ pertenece $a$ $D(T)$. Además $(T+i) h(x)=(x+i) h(x)=f(x)$. Por 10 tanto $\operatorname{Ran}(T+i)=\mathcal{L}^{2}(\mathbb{R})$. Un argumento similar demuestra que Ran $(T-i)=$ $=\mathscr{L}^{2}(\mathbb{R})$. Aplicando el teorema anterior se deduce que $\mathrm{T}$ es autoadjunto.

Ejemplo. (Hamiltoniano). El operador de Energía o Hamiltoniano está definido por

$$
H=\frac{1}{2 m} L^{2}+V=-\frac{h^{2}}{2 m} \frac{\partial^{2}}{\partial x^{2}}+V(x)
$$

donde $\mathrm{V}$ es una función contínua que satisface

$$
\int_{\mathbb{R}}|\mathrm{V}(\mathrm{x})|^{2}|\mathrm{f}(\mathrm{x}, \mathrm{t})|^{2} \mathrm{dx}<\infty .
$$

Definimos $D(V)=\left\{f \in \mathcal{L}^{2}(\mathbb{R})\right.$ : $\mathrm{f}$ satisface $\left.(I)\right\}$ y denotemos por $L$ el operador diferencial $L=i h \frac{\partial}{\partial x}$ donde $h$ representa una constante. De finamos $H_{0}$ el operador dado por $H_{0}=\frac{1}{2 m} L^{2}$. Formalmente podemos escribir

$$
\mathrm{H}=\mathrm{H}_{0}+\mathrm{V} \text {. }
$$

Para darle un sentido a esta última expresión debemos definir los dominios 
correspondientes de $\mathrm{H}_{0}$ y del operador multiplicación por $\mathrm{V}$. Para este último tomamos $\mathrm{D}(\mathrm{V})$. Usando la transformada de Fourier en $\mathscr{L}^{2}(\mathbb{R})$ (la cual es un operador unitario de $\mathcal{L}^{2}(\mathbb{R}) \stackrel{\wedge}{\longrightarrow} \mathcal{L}^{2}(\mathbb{R})$ ) vemos que el operador $\mathrm{L}$ es unitariamente equivalente a multiplicación por $\mathrm{x}$ en $\mathfrak{L}^{2}(\mathbb{R})$. Además dejamos como ejercicio al lector probar que si $L$ es autoadjunto entonces $L^{2}$ también lo es. En forma similar al ejemplo anterior se demuestra que $L$ es autoadjunto en el dominio $D(L)$

$$
D(I)=\left\{f \in \mathcal{L}^{2}(\mathbb{R}): \int_{\mathbb{R}} x^{2}|f(x, t)|^{2} d x<\infty\right\}
$$

Resulta natural tomar $\mathrm{D}\left(\mathrm{H}_{0}\right)=\mathrm{D}\left(\mathrm{L}^{2}\right)$. Notemos que el operador $\mathrm{H}=\mathrm{H}_{0}+\mathrm{V}$ está definido en $\mathrm{D}\left(\mathrm{H}_{\mathrm{o}}\right) \cap \mathrm{D}(\mathrm{V})$.

Finalizamos el estudio de este operador dando condiciones sobre el potencial $V(x)$ para que $H$ sea autoadjunto.

Teorema 1.13. Si existe a $<1$ tal que

$$
\|v \psi\| \leq \mathrm{a}\left\|\mathrm{H}_{0} \psi\right\|+\mathrm{b}\|\psi\|
$$

para todo $\psi$ en $D\left(H_{0}\right)=D\left(L^{2}\right)$ entonces $H$ es autoadjunto.

Demostración: Ver [4] volumen II.

Ejemplo. Si el potencial $\mathrm{V}(\mathrm{x})$ tiene la propiedad de que

$$
\sup _{x} \int_{x}^{x+1}|v(y)|^{2} d y<\infty
$$

entonces $V$ satisface la desigualdad del teorema 1.13. 
§ 2. Teoría espectral.

En esta sección generalizaremos el teorema espectral (ver Capítulo I, teorema 5.6.). Las definiciones dadas en la sección 4 del primer Capí tulo son esencialmente las mismas para el caso no acotado, teniendo cuida do con los dominios de los operadores. Nos interesarán solamente los ope radores cerrados.

Definición 2.1. Sea $T$ un operador lineal cerrado en $H \cdot \lambda$ es un punto del resolvente $\rho(T)$ si $\lambda I-T$ es una biyección de $D(T)$ en $H$ que posee una inversa acotada, es decir, existe $\mathrm{B}: \mathrm{H} \rightarrow \mathrm{H}$ tal que

$$
B(\lambda I-T)=I_{D}(T) \quad Y \quad(\lambda I-T) B=I_{H} .
$$

El espectro $\sigma(T)$ se define de la misma manera que para el caso acotado. La demostración del teorema siguiente es similar al del caso acotado (ver Capítulo I, teorema 4.2.).

Teorema 2.2. Sea $T$ un operador lineal cerrado densamente definido en $H$. Entonces

i) $\quad \rho(T)$ es abierto

ii) $\quad R_{\lambda}(T)-R_{\mu}(T)=(\mu-\lambda) R_{\mu}(T) R_{\lambda}(T)$.

Muchas propiedades importantes de los operadores no acotados son muy sensibles a los cambios de dominio. El siguiente ejemplo muestra que el espectro es una de esas propiedades. 
Ejemplo: Denotemos por $\mathrm{AC}[0,1]$ a las funciones absolutamente contínuas en $[0,1]$ cuyas derivadas son de cuadrado integrable. Sea $T_{1}=T_{2}=i \frac{d}{d x}$ con dominios

$$
\begin{aligned}
& D\left(T_{1}\right)=A C[0,1] \\
& D\left(T_{2}\right)=\{f \in A C[0,1]: f(0)=0\}
\end{aligned}
$$

Estos dominios son densos en el espacio de Hilbert $\mathcal{L}^{2}[0,1]$ y es un bonito ejercicio de análisis demostrar que $\mathrm{T}_{1}$ y $\mathrm{T}_{2}$ son operadores cerrados. Probaremos que $\sigma\left(\mathrm{T}_{1}\right)=\mathbb{C}$ y que $\sigma\left(\mathrm{T}_{2}\right)=\phi$. Es claro que para todo $\lambda \in \mathbb{C}$ existe $f(x)=e^{-\lambda x i}$ en $D\left(T_{1}\right)$ tal que

$$
\left(\lambda I-T_{1}\right)=0 .
$$

Por 10 tanto $\left(\lambda I-T_{1}\right)$ no puede ser invertible para todo $\lambda \in \mathbb{C}$, en otras palabras, $\sigma\left(\mathrm{T}_{1}\right)=\mathbb{C}$.

Para demostrar que $\sigma\left(\mathrm{T}_{2}\right)=\phi$ basta ver que $\lambda I-\mathrm{T}_{2}$ es una bi yección entre $D\left(T_{2}\right)$ y $H$ puesto que por el Teorema 1.4. la inversa será automáticamente acotada. Probar que $\operatorname{Ker}\left(\lambda I-T_{2}\right)=\{0\}$ es equivalente a encontrar soluciones en $\mathrm{D}\left(\mathrm{T}_{2}\right)$ de la ecuación diferencial homo génea

$$
\left\{\begin{array}{r}
\lambda y-i y^{\prime}=0 \\
y(0)=0
\end{array}\right.
$$

Este problema de valor inicial tiene una única solución, la función idénticamente nula 
Probar que $\lambda I-T_{2}$ es sobre es equivalente a encontrar una solución $f$ en $\mathrm{D}\left(\mathrm{T}_{2}\right)$ del problema del valor inicial no homogéneo.

$$
\left\{\begin{array}{r}
\lambda y-i y^{\prime}=g \\
y(0)=0
\end{array}\right.
$$

para $g$ en $\mathscr{L}^{2}(\mathbb{R})$. Este último problema tiene una única solución dada por

$$
f(t)=i e^{-i \lambda t} \int_{0}^{t} g(x) e^{i \lambda x} d x
$$

Es fácil demostrar que $\|f\|_{\mathcal{L}^{2}[0,1]} \leq \sqrt{2}\left\|_{g}\right\|_{2}$ y claramente, por ser solución de la ecuación, se tiene que $f(0)=0$ y además $f^{\prime}$ existe y es igual a $(i g-i \lambda f) \in \mathcal{L}^{2}(\mathbb{R})$, luego $f$ es absolutamente contínua con derivada en $\mathcal{L}^{2}(\mathbb{R})$.

Ahora daremos la versión del teorema 5.6. para el caso de operadores no acotados.

Teorema 2.3. Sea $\mathrm{T}$ un operador densamente definido y autoadjunto en $\mathrm{H}$; H espacio de Hilbert separable. Entonces existen un espacio de medida $(M, \mu)$ con $\mu(M)$ finito, un operador $U: H \rightarrow \mathcal{L}^{2}(M, \mu)$ Y una función $f$ definida en $M$ a valores reales finita en todas partes tal que
a)
$\psi \in \mathrm{D}(\mathrm{T})$ sí y sólo si $f(\cdot)(\mathrm{U} \psi)(\cdot) \in \mathcal{L}^{2}(M, \mu)$
b)
Si $\phi \in U[D(T)]$, entonces $\left(\mathrm{UTU}^{-1} \phi\right)(\mathrm{m})=\mathrm{E}(\mathrm{m}) \phi(\mathrm{m})$. 
Demostración: Por teorema 1.12. del segundo Capítulo sabemos que $\operatorname{Ran}(T \pm i)=H \quad$ y que $\operatorname{Ker}(T \pm i)=\{0\}$. Por 10 tanto $(T \pm i)$ poseen una inversa. Estas inversas resultan ser acotadas por el teorema 1.4. y del hecho que $T$ es cerrado. Además aplicando el teorema 2.2. de esta sección se tiene que $R_{i}(T)$ y $R_{-i}(T)$ conmutan. La igualdad

$$
\left\langle(T-I) \psi,(T+i)^{-1}(T+i) \phi\right\rangle=\left\langle(T-i)^{-1}(T-i) \psi_{r}(T+i) \phi\right\rangle
$$

junto con $\operatorname{Ran}(T \pm i)=H$ prueba que $\left((T+i)^{-1}\right)^{*}=(T-i)^{-1}$. En resumen hemos demostrado que $(T+i)^{-1}$ es normal.

Aplicando el teorema 5.6. a $(T+i)^{-1}$ se tiene que existe un espacio de medida finita $(M, \mu)$, un operador unitario $U: H \rightarrow \mathcal{L}^{2}(M, \mu)$ y una función $g$ a valores complejos definida en $M$, $g$ acotada tal que

$$
\left(U(T+i)^{-1} U^{-1} h\right)(m)=g(m) h(m)
$$

para todo $h$ en $\mathcal{L}^{2}(M, \mu)$.

Puesto que $\operatorname{Ker}(T+i)^{-1}$ es vacío entonces $g(m) \neq 0$ casi en todas partes (c.t.p.) . Luego $[\mathrm{g}(\mathrm{m})]^{-1}$ existe como función y es finita $\mu$ - c.t.p. Definamos $f(m)=[g(m)]^{-1}-i$

a) Sea $\psi \in \mathrm{D}(\mathrm{T})$. Entonces existe $\phi$ en $\mathrm{H}$ tal que $(T+i)^{-1} \phi=\psi$ Por 10 tanto tenemos que

$$
U \psi=U(T+i)^{-1} \phi=\left(U(T+i)^{-1} U^{-1}\right)(U \phi)=g(U \phi)
$$

$y$ entonces $f(\bullet)(U \psi)(\cdot)=f(\bullet) g(\bullet)(U \phi)(\cdot)$. 
Como fg es acotada y $\mathrm{U} \phi \in \mathcal{L}^{2}(M, \mu)$ se obtiene que $f(\cdot)(U \psi)(\cdot)$ es un elemento de $\mathcal{L}^{2}(M, \mu)$.

Supongamos ahora que $f(\cdot)(U \psi)(\cdot)$ está en $\mathscr{L}^{2}(M, \mu)$. Entonces como $U$ es un isomorfismo existe $\phi \in H$ tal que $U \phi=(f+i) U \psi$. Por 10 tanto

$$
g(U \phi)=(g)(f+i) U \psi=U \psi
$$

Además

$$
g(U \phi)=\left(U(T+i)^{-1} U^{-1}\right)(U \phi)=U(T+i)^{-1} \phi
$$

Comparando estas identidades obtenemos $\quad U(T+i)^{-1} \phi=U \psi$ lo cual demuestra que $\psi \in D(T)$.

b) Sea $\psi \in D(T)$ entonces existe $\Phi \in H$ tal que $\psi=(T+i)^{-1} \phi$ y $T \psi=\phi-i \psi$. Por 10 tanto

$$
\begin{aligned}
(U T \psi)(m) & =U(\Phi-i \psi)(m) \\
& =(U \Phi)(m)-i(U \psi)(m) .
\end{aligned}
$$

A partir de $\psi=(T+i)^{-1} \phi$ deducimos lo siguiente

$$
\begin{aligned}
U \psi=U(T+i)^{-1} \phi & =U(T+i)^{-1} U^{-1}(U \phi) \\
& =g(U \phi) .
\end{aligned}
$$

Por 10 tanto como $\mathrm{g}(\mathrm{m}) \neq 0$

$$
(U T \psi)(m)=\left([g(m)]^{-1}-i\right)(U \psi)(m)
$$




$$
=f(m)(U \psi)(m) \text {. }
$$

Finalmente, puesto que $U$ es un operador unitario $y \quad \sigma(T)$ es un subconjunto de $\mathbb{R}$ se tiene que $f$ es una función a valores reales.

\section{\$ 3. Comentarios finales.}

Hemos visto en este cursillo que los operadores autoadjuntos y normales tanto acotados como no acotados son unitariamente equivalentes a operadores multiplicación en algún espacio de Hilbert $\mathcal{L}^{2}(\mathrm{M}, \mu)$. Los operadores multiplicación se pueden estudiar desde varios puntos de vista: por ejemplo, dados dos operadores multiplicación $\mathrm{T}_{\phi}, \mathrm{T}_{\psi}$, ¿cuándo son ellos unitariamente equivalentes? Para el lector que se interese en este apasionante problema recomendamos el artículo de Abrahamse "Multiplication operators" (Lecture Notes \# 693, Springer-Verlag (1973), 17-36). Otra pregunta interesante es, ¿cómo generalizar el concepto de multiplicidad para operadores multiplicación? Así los operadores $T_{x}$ y $T_{x^{2}}$ tienen multiplicidad uno y dos respectivamente. El artículo de Abrahamse y Kriete "The spectral multiplicity of a multiplication operator" (Indiana University. Math. Journal 22 (1973), 845-857) da una visión del problema.

Finalmente, desde el punto de vista de la Matemática física, el estudio de los operadores de ondas generalizadas definidos por

$$
\Omega(A, B)=s-\lim _{t \rightarrow \pm \infty} e^{i A t} e^{-i B t} P_{A C}(B)
$$


para $A, B$ operadores autoadjuntos y de donde $P_{A C}(B)=P_{H_{A C}}(B)$; resul tan de interés en Matemática, debido a que son operadores unitarios que poseen propiedades interesantes y son muy usados en la Teoría de Pertur baciones. Unas excelentes referencias para este tópico es [4], volúmenes III y IV y Kato [2] .

BIBLIOGRAFIA.

[1] Douglas, R.: "Banach Algebras Techniques in Operator Theory" Academic Press, New York (1973).

[2] Kato, T. : "Perturbation Theory for Linear Operators" Springer-Verlag, New York (1966).

[3] Rudin, W. : "Functional Analysis" Tata-Mc Graw-Hill

[4] Reed-Simon.: Volume I "Functional Analysis" Volume II "Fourier Analysis, selfadjointness" Volume III "Scattering Theory" Volume IV "Analysis of Operators" Academic Press, New York (1975) 